\title{
"Problemas psicosociales que viven los estudiantes con orientación homosexual y bisexual de la ESAc"
}

\author{
"Psychosocial problems experienced by students with homosexual and bisexual orientation of \\ the ESAc" \\ Maria. E. Gomez-Gamero ${ }^{a}$
}

\begin{abstract}
:
The purpose of this research was to identify the problems of the students with homosexual and bisexual orientation in different degrees in Escuela Superior de Actopan (ESAc). The facts who probably alter your physical health and emotional. In the accept process of the sexual orientation interview different facts to accept with their families, friend groups, or the context with they stay socially and culturally, in this process victimization can be present by their election, discrimination with family or classmate, they feel isolation, alone, frustration, impotently, and the consequences of this feelings, can be presented as anxiety disorder, depression disorder, or even the risk of committing suicide.
\end{abstract}

\section{Keywords:}

Sexual orientation, acceptance, physical and emotional health

\begin{abstract}
Resumen:
El presente trabajo de investigación tuvo la finalidad de identificar los problemas a los que se enfrentan en la vida los alumnos con orientación homosexual y bisexual inscritos en las diferentes carreras de la Escuela Superior de Actopan (ESAc). Factores que pueden alterar su salud física como emocional. En el proceso de la aceptación sobre la orientación sexual intervienen diferentes factores para la aceptación dentro de su familia, con el grupo de amigos, o en el contexto en donde se desenvuelven social y culturalmente, en este proceso la victimización puede estar presente por su elección, así como la discriminación de parte de familiares o compañeros de clase, que comporta sentimientos de aislamiento, soledad, frustración e impotencia y las consecuencias de estos sentimientos, se pueden presentar como trastorno de ansiedad, depresión o incluso el riesgo de cometer un suicidio.
\end{abstract}

\section{Palabras Clave:}

Orientación sexual, aceptación, salud física y emocional

\section{Introducción}

La homosexualidad es un fenómeno social complejo aún, sus connotaciones siguen siendo conflictivas para una sociedad de hegemonía patriarcal y arraigos machistas, a pesar de los avances globales. (Uribe, M., Pérez, E., y Arotoma, R., 2018).

La sociedad recientemente ha estado hablando sobre cómo se vive y se piensa sobre la "homosexualidad y bisexualidad", durante mucho tiempo las diferencias entre la sexualidad masculina y femenina han tratado de ser explicadas desde lo biológico, esto depende de los comportamientos y actitudes que se deben asumir, de acuerdo al sexo que se asignó al nacer, estas divisiones se han interiorizado desde la infancia y se asumen como roles establecidos socialmente, en donde las personas están obligadas a cumplir las normas que impone la sociedad; roles en los que se asigna a la mujer como ama de casa y al hombre como figura de autoridad, la mayoría social determina el rol que deben desempeñarse, los estereotipos adquieren fuerza que no serían benéficos para la inclusión de las personas en una sociedad patriarcal.

En una sociedad heterosexista y machista, es más aceptable un homosexual masculino que un homosexual femenino, por los comportamientos propios de un género que se visibiliza y se reafirma socialmente. La depresión y ansiedad son problemas de salud mental que sobresalen en la construcción de esta identidad en el momento de la elección de la orientación sexual (Granados, J, Y Delgado, G., 2008), las experiencias de aislamiento, devaluación personal, discriminación, violencia verbal y física en muchas ocasiones tienen su origen por los prejuicios y creencias culturales, y se tiene como 
consecuencia el ocultamiento de la orientación sexual que implica también el miedo a la censura, al rechazo familiar y social cuando se decide desvelar la orientación sexual (la homosexualidad o bisexualidad).

En México perdura la cultura de la sexualidad dominante del heterosexismo, como parte de esta cultura, el machismo y la homofobia subsisten (Piña, J., \& Aguayo, H., 2015) esto hace que se vea a la heterosexualidad como la base de las normas de la sexualidad. La discriminación hacia los homosexuales en México tiene lugar desde la época de la prehistoria, donde los aztecas condenaban violentamente su conducta (Lazano, 2009). La consecuencia de esto es que, a lo largo del tiempo, a los homosexuales se les cataloga de forma negativa, se les ridiculiza, se les teme o se les agrede (Piña, J., \& Aguayo, H., 2015), como ejemplo de la agresión, en 2011 en la Ciudad de México se registraron denuncias del asesinato de 35 personas de la comunidad LGBT y asi posteriormente se siguen presentando. En México se distinguen aun las prácticas de discriminación, exclusión y crímenes de odio por homofobia, dando como resultado que la discriminación sea el principal problema que enfrentan las personas con orientación homosexual, y el segundo la falta de aceptación, y la aparición de críticas y burlas (González, R., \& Valles, R., 2010).

De igual manera, otro de los problemas a los que se enfrentan las personas con orientación homosexual 0 bisexual es la estigmatización, convirtiéndose en factor que genera en el individuo con esta orientación bajo nivel de autoestima y en la que se incrementa la susceptibilidad a enfermedades mentales, como la depresión y ansiedad. Estas condiciones conllevan a que algunos integrantes de esta comunidad vivan su vida sexual como un conflicto psíquico que determinará las formas en que se desenvuelva en otros ámbitos.

Por otra parte, la aceptación de los padres es de fundamental importancia, ya que estos constituyen la principal red de apoyo a lo largo de su vida, de este modo, si los padres reaccionan de una manera negativa ante la confesión de la decisión sobre la orientación sexual de algún miembro de la familia, podría afectar al proceso de afrontamiento $e$ incluso ante otros sectores de la sociedad.

La actual controversia que existe sobre la diversidad de la orientación sexual, se ha distinguido a lo largo del tiempo y alrededor del mundo se han dado diferentes movimientos sobre la libertad de la expresión de la sexualidad, en donde sujetos dan a conocer su inconformidad sobre las pasadas formas de orden social, en las que implican a la sexualidad que aun en nuestros tiempos se asocia a su aceptación social que tienen su origen en las causas que llevan a realizar dichos movimientos y lo que se busca obtener con ello.

La presente tiene un enfoque de investigación cuantitativo, el objetivo se determinó en recolectar datos que nos describan los problemas sociales que viven los estudiantes con orientación homosexual y bisexual de las licenciaturas de Psicología y Diseño Gráfico de la Escuela Superior de Actopan (ESAc), señalando que no se ha tenido relevancia en este tema y en esta comunidad, nos encontramos en un campo, que no cuenta con trabajos de investigación sobre el tema, se desconocen los problemas que viven los jóvenes con esta orientación sexual en nuestra institución. De este modo, el principal interés es descubrir e identificar los principales problemas sociales por los que atraviesan e identificar si las normas que dicta la sociedad con respecto a la sexualidad son el principal factor para que estas personas repriman su sexualidad y no la den a conocer. El aporte es un punto de vista más amplio sobre las circunstancias que viven las personas al tratar de buscar una inclusión social. La homo-bi-fobia se expresa tanto de manera física como verbal e identificar si es menos marcada entre los universitarios, se lleva a cabo la investigación comparando las similitudes y diferencias entre los estudiantes de la Licenciatura en Diseño Gráfico y Psicología.

\section{Eventos pasados}

La homosexualidad existe, desde épocas remotas y hablar del sexo se presenta como un tema de tabú dentro de la sociedad. La comunidad homosexual y bisexual desde épocas históricas han pasado por situaciones de discriminación, ejemplo de esto se puede citar a personas que se han condenado a muerte, que han sido masacrados o perseguidos. Durante la prehistoria existieron dos etapas que reflejan la perpetuación de la especie como: la monogamia natural y la monogamia con finalidad de asegurar el patrimonio familiar. En la Edad Antigua no se aplicaba el término de homosexual, era más acertado hablar de "prácticas sexuales", ya que lo más corriente era la bisexualidad, el juicio social hacia los actos sexuales no se basaba en la orientación sexual o el género, la única diferencia era en la sexualidad, el rol de activo (predominante en el varón) o pasivo (asignado solamente a la mujer). En Mesopotamia se registra la existencia de algunos sacerdotes de Ishtar homosexuales, que participaban bailando travestidos en determinados ritos, se presentaba en gran cantidad la prostitución masculina. Sin en embargo, en Egipto son muy escasos y la mayoría de las veces ambiguos, aquí el incesto estaba permitido y la circuncisión, tenía un carácter ritual en la adolescencia (Vera, L., 1998). 
En Grecia aparecieron los primeros escritos de prácticas homosexuales; la pederastia homosexual masculina era una costumbre muy arraigada, la relación empezaba cuando el amante adulto estaba en la veintena y el chico entrando a la pubertad, contraían matrimonio cuando el adulto tuviese treinta, no con fines sexuales sino como el rol de tutor responsable de la educación y entrenamiento militar del joven. Entre los griegos se tenía la creencia que el semen era fuente de conocimiento y teniendo relaciones pasaban la sabiduría que se transmitía de una generación a otra y se aceptaba esta orientación sexual, existieron otras civilizaciones y concepciones, como la que incluía la prostitución y sexo con esclavos, también el lesbianismo se presentaba, pero en menor cantidad. De igual manera, en Roma se practicó la prostitución masculina y existieron los primeros matrimonios gay con contrato privado donde no intervenía el Estado. Desde mediados de la Época Imperial fue prohibida la orientación sexual homosexual en la Era Cristiana y se castigaba con pena de muerte. En Asia Oriental, la atracción por el mismo sexo se consideró normal y no perseguida como en Europa durante la Edad Media (Vera, L., 1998).

Anteriormente no se trataba al homosexual o bisexual como criminal sino como enfermo, sin embargo, en 1973, la APA lo excluyo como enfermedad mental en la clasificación del DSM, para Cabaj y Stein (1996) quiénes recalcan que no era una enfermedad mental y diez años después se eliminó todo diagnostico psiquiátrico que pudiera ser usado para discriminar a la comunidad homosexual.

En los 70's se organizan los grupos de activistas debido a que se despide a un empleado de una compañía transnacional por su conducta homosexual, este suceso reunió a varios grupos de la comunidad LGBT quiénes cuestionaron la estigmatización y opresión social. A partir de ello se llevó a cabo la primera Marcha del Orgullo Homosexual en México en el año de 1980 (Lazano, 2009). Estas marchas siguen realizándose y anualmente, el 28 de julio, se lleva a cabo con la finalidad de promover una mayor defensa a sus derechos humanos, al igual que la pertenencia a una sociedad incluyente e igualitaria fuera de todo acto discriminatorio, así como el respeto a la libre decisión para integrar parejas del mismo sexo, considerando la defensa de la identidad sexual, la identidad de género y la orientación sexual. Actualmente se han unido más personas a la comunidad LGBT que paso a nombrarse LGBTTTQI (Lesbianas, Gays, Bisexuales, Transexuales, Transgénero, Travestis, Queer e Intersexuales), también es notorio que las amenazas verbales y físicas han disminuido, pero la incomodidad puede notarse en escenas de la vida cotidiana al ser observados y aún más, si la preferencia sexual de la persona es muy evidente. Nuestra actual sociedad muestra respeto ante estas situaciones, si esto no afecta su entorno cotidiano sin embargo no es algo que se apruebe, y se toman conductas como ignorarlos o no hablarles.

\section{Iniciando en la familia}

La familia se ha considerado como la célula fundamental en la sociedad y establece las bases para la reproducción de los valores y la permanecía de las estructuras sociales. De igual manera, la familia es la encargada de la educación afectiva, sexual y de la preparación para la incorporación de los individuos en los diferentes ámbitos sociales. De este modo, Careaga y Salvador (2004) concluyen que la familia es la responsable del "buen comportamiento" y del futuro de cada uno de sus miembros. A su vez, la familia no trabaja aislada en la educación de sus integrantes, en muchas ocasiones se apoya de los recursos del Estado para cumplir con su quehacer. La familia dicta sus normas a cada individuo, establecerá el rol y el lugar social donde se va a instaurar, buscado definir la identidad, la orientación y las perspectivas aceptables para cada uno de sus miembros. Por ello, a la familia se establece como símbolo de autoridad, en donde se restringe y ordena a los miembros de acuerdo a sus propios intereses. Cuando la instancia de la familia se encuentra con algo que esta fuera de los límites de lo aceptado, se encuentra ante una amenaza, y recurre a los elementos que la sociedad le ofrece, como el silencio y la negación, también pueden buscar apoyo de instancias más adecuadas para arreglar estos "problemas" (Careaga, 2004).

Con relación a la sexualidad, a lo largo del tiempo se ha visto restringida al ámbito conyugal y con fines reproductivos, el reconocer la sexualidad fuera del entorno familiar produce una estigmatización a quién la ejerce, empeorando cuando no se sigue la tradicional estructura reproductiva de la familia. De este modo, se podría concluir que la familia no ha tenido un control completo de la expresión sexual de sus integrantes, lo que ha generado un sentimiento de inconformidad, rechazo y hostilidad hacia quiénes se expresan de modo diferente (Careaga, 2004). Para que la familia pueda mantener un orden sobre la expresión sexual, en ocasiones se hace uso de la violencia. Se distingue al silencio, la negación como las principales formas de violencia y ocultamiento, sin embargo, en algunas familias se normaliza el abuso sexual a menores, las relaciones incestuosas y la violencia en todas sus formas de expresión hacia los integrantes de la familia.

Con respecto a la sexualidad diferente a la heterosexualidad, se mantiene regulada mediante la negación, se niega lo que se es, y se considera que una relación homosexual es sucia, enferma, perversa, pecaminosa y equivocada. Esto genera en los sujetos un 
distanciamiento social, tanto de los otros integrantes de la familia como de la sociedad por ser considerados como "diferentes". Viven con la orientación sexual que no es heterosexual y su sexualidad es una contradicción que va desde el orgullo a la culpa causada por la moral social (Careaga, 2004). Para Salvador (2004) señala que compartir el secreto es más fácil entre iguales que con los familiares que se consideran una figura de autoridad, por este motivo existe el sentimiento de temor a la estigmatización, a la desaprobación y a la violencia que puedan surgir. Compartir este secreto a las amistades se hace dependiendo de la aceptación ante este fenómeno, el lazo afectivo no es primordial para la posibilidad de apertura. La aceptación de la familia, de la pareja, se puede decir que es muy limitado que esto se permita. Sin embargo lo aceptado socialmente y en la familia son la relaciones heterosexuales en las que se pueden compartir diversos momentos familiares, a diferencia de las personas homosexuales que se limitan a convivir con su pareja en situaciones difíciles o en un pequeño círculo y esto hace que se fortalezca la estigmatización (Careaga, 2004).

\section{La discriminación}

Las personas que, por su vestimenta, actitudes e interacciones, muestran con mayor facilidad su condición sobre la diversidad sexual, no solo sufren la discriminación y el rechazo por parte de la familia, de los amigos, se enfrentan también a la discriminación social, al ser observados, señalados y excluidos de los grupos sociales que perduran dentro de la sociedad y que rechazan a las personas homosexuales incluso más que a las bisexuales. Estas condiciones de rechazo pueden generar en el individuo, que ellos mismos no se acepten y de igual forma por su condición de diferencia (Careaga, 2004). En el 2015, Piña y Aguayo señalan que la homofobia se debe en gran parte a que la homosexualidad atenta contra grandes instituciones políticas y sociales, como el matrimonio y la familia. Así mismo, la comunidad LGBT ha exigido el derecho a ser reconocidas por dichas instituciones. Las personas homosexuales y bisexuales por mucho tiempo han sido blanco fácil de la sociedad, cuyas ofensas vienen de personas heterosexista y machistas manifestándose en el acoso, hostilidad, discriminación, rechazo y violencia, llevándolos a situaciones de depresión, aislamiento, desesperación y finalmente han desencadenado en suicidio así mismo en homicidios. "Aún hay muchas personas que creen que la homosexualidad "no es normal" y que por esa razón debe tratarse, corregirse o reorientarse con "terapias de reconversión" que representan una amenaza a sus propias vidas, a sus derechos y a un deterioro emocional. Igualmente, la estigmatización causa un serio impacto en las relaciones interpersonales, interacciones sociales y en la autoestima de muchos homosexuales" (OPS/OMS, 2011).

\section{La orientación sexual en México}

En nuestro país, una de las principales representaciones de discriminación se enfoca hacia la preferencia sexual, en poblaciones rurales e indígenas existen menos estudios, los indígenas homosexuales, no existen ni para el sector público, ni para el Estado (Fregoso, 2018). Para Mondragón (2018) las personas indígenas por el solo hecho de serlo, son objeto de racismo cotidiano, de este modo, los indígenas que son homosexuales poseen ya una coraza y menor vulnerabilidad a los ataques de racismo, sin embargo, se violentan sus derechos. De la misma manera, las costumbres y tradiciones en sus comunidades, crean en ellos un sentimiento de temor al ser rechazados y expulsados de la misma. Estos son los principales factores por los que se oculta la existencia de integrantes homosexuales en comunidades indígenas. Por otra parte, a las etnias se les ha infantilizado para que no piensen en su sexualidad (Fregoso, 2018). En las zonas urbanas las condiciones de las personas con una orientación sexual diferente a la heterosexualidad, se vive de manera distinta a las de las personas que viven en zonas rurales. Los principales problemas que enfrentan son la discriminación en los ámbitos laborales, al caminar por la calle a pesar de que nos son insultados, ni violentados, si son observados de manera despectiva. Sin embrago uno de los logros que se ha obtenido en la Cuidad de México es el hecho de aprobar el derecho al matrimonio igualitario (Redondo, 2017).

\section{Desde la teoría Freudiana}

El término bisexual no resulta nuevo en la sociedad, los casos más notorios se presentaron desde las teorías del psicoanálisis de Sigmund Freud, quién aseguro que "todos en potencia somos bisexuales", es decir, el que nos guste un determinado sexo es porque la sociedad nos lleva a inclinarnos hacia ello, dependiendo si nacemos hombre o mujer. Freud plantea, que, si un ser humano desde su nacimiento no se le inculca, por naturaleza le gustaran ambos sexos, él fue el primero en estudiar la bisexualidad humana. Probablemente si un heterosexual decide "experimentar" con alguien de su mismo sexo es posible que le guste, no solo como algo placentero sino emocionalmente. Esto se asocia con la Teoría Freudiana de la Personalidad, en donde en el "Ello" se presentan los deseos e impulsos, el "Yo" es visto como la realidad del mundo y en el "Superyó" se incluye la moral, la ética, las costumbres religiosas y/o familiares (Tabasco, 2013). Para Freud la orientación sexual como un objeto de enamoramiento y la elección homosexual o heterosexual se definen a partir de la resolución de la conflictiva edípica 
en todos los seres humanos, llamado "Complejo de Edipo", que define como el deseo inconsciente de mantener una relación sexual con el progenitor del sexo opuesto y de eliminar al padre del mismo sexo. De modo que, el complejo de Edipo, positivo (amor al progenitor del sexo opuesto) o negativo (amor al progenitor del mismo sexo), marca el momento en el cual la sexualidad parcial y las pulsiones parciales se reunifican en el amor al otro como objeto total (Gasque, 2016).

\section{Proceso para desvelar la orientación sexual}

La construcción de identidad sexual es un proceso en el cual se adquiere la conciencia y se consolida la orientación sexual (Jiménez, A., y Romero, M., 2014). Esta se va desvelando desde la infancia, cuando los progenitores a través la propia educación contribuyen con la enseñanza de los términos de que lo que significa ser un hombre y mujer. Estas enseñanzas en la mayoría de los casos son consideradas como un duelo heterosexista, que es diferente en cada cultura, familia y tradición (Vitalit \& Onofrio, 2011). De este modo, las personas que no se identifican con la identidad sexual heterosexual se encuentran en un proceso de reintegración, el primer paso es la aceptación propia, en un proceso para reconocer que se tiene una identidad sexual diferente a lo establecido por la familia, pero este proceso es largo y difícil y las personas comienzan a descubrir la diferencia de su sexualidad (Vitalit \& Onofrio, 2011).

Para Troiden (1989) la identidad homosexual es de autoidentidad se muestra cuando estas personas se ven como un individuo gay o lesbiana. En el modelo de Pérez (2005) propone el proceso de la construcción de la orientación sexual que está ligada con el proceso de identidad sexual, que se presenta en 5 etapas:

1. Sensibilización, donde se identifican los deseos homoeróticos que provocan confusión.

2. Conciencia, donde se reconoce el objeto del deseo, se supone como algo pasajero.

3. Autodefinición, donde ellos se autodenominan homosexuales.

4. Aceptación, reorganización de las jerarquías del "Yo", aparecer como orgullo por ser diferente y se exige un cambio social.

5. Integración, poseen estrategias para protegerse contra el rechazo social (Jiménez, A., y Romero, M., 2014).

\section{Inclusión de la diversidad sexual}

La inclusión social de un individuo con orientación sexual diferente, se lleva a cabo desde el hogar, en su entorno familiar lugar en el que se desenvuelve con cada uno de sus integrantes, cuanto mayor apoyo brinde la familia, mayor herramientas personales y sociales tendrá una persona homosexual o bisexual para afrontar los obstáculos que se interpondrán en su vida, a diferencia de un homosexual rechazado por su familia, que estará solo para enfrentar a la sociedad, mediante sus propios medios y sin apoyo (Careaga, 2004), aunque posiblemente, el individuo se deje vencer por la soledad, el alcohol, el tabaco o por sustancias dañinas que por ende provoquen problemas mentales y físicos en ellos. En la actualidad, en algunos países, se ha mejorado la actitud de conciencia hacia los temas de diversidad sexual, en el que se han reconocido los derechos, como derechos universales. En muchos otros países es ilegal ser gay, y se les castiga por ello, con respecto a los derechos y la libertad de expresión, son reprimidos o se les violenta y el trato que reciben las personas homosexuales, bisexuales, en estos países es grotesco e inadecuado (Richaed, 2013).

Para los funcionarios de las Naciones Unidas que se han visto interesados en los actos de violencia hacia los derechos humanos de las personas LGBT. Se están organizando y han incorporado a sus planes de trabajo los problemas principales que viven estas personas estableciendo que el no respetar sus derechos y no proteger a las personas LGTB de los abusos y discriminación, supone una violación de las normas internacionales de los derechos humanos. Es necesario tomar en cuenta lo establecido por estas organizaciones para generar la inclusión de las personas LGTB, ya que de lo contrario nos enfrentamos a una violación de estas normas y se pueden generar respuestas negativas como la vulnerabilidad a enfermedades, a infecciones por VIH y a la exclusión social y económica (PND/PGA, 2017).

\section{Metodología de Investigación}

Nuestra investigación tiene un enfoque cuantitativo, exploratorio y transversal. Para la muestra se tomó en cuenta al universo de la población que es de 312 estudiantes en la Licenciatura de Psicología más 430 de la Licenciatura en Diseño Gráfico, la muestra mínima se establece para estudios cuantitativos es de 30 personas, sin embargo la población sujeta a esta estudio es menor participando 15 alumnos en el estudio. De ellos el $60 \%$ $(n=9)$ son mujeres y el $40 \%(n=6)$ son hombres, que se encuentran matriculados en la Escuela Superior de Actopan en ambas licenciaturas y el rango de edad oscila entre los 18 y 28 años de edad.

Las licenciaturas que participan son Psicología con un $40 \%(n=6)$ de los participantes y Diseño Gráfico con un $60 \%(n=9)$, la participación estudiantil se encuentra en diferentes semestres, en segundo semestre existe un porcentaje del $13.3 \%(n=2)$, en tercero $40 \%(n=6)$, en cuarto $20 \%(n=3)$, en quinto $13.3 \%(n=2)$ y por último en octavo con $13.3 \%$ ( $n=2)$. La mayoría de ellos provienen del Estado de Hidalgo, representando $73.5 \%$ de la 
muestra total $(n=11)$, mientras que el $26.8 \%(n=4)$ son del Estado de México. De la muestra el 6.7\% $(n=1)$ declararon ser lesbianas, asimismo $33.3 \%(n=5)$ gays y el $60 \%(n=9)$ son bisexuales, visto como la orientación sexual con mayor rango de porcentaje.

\section{Instrumento de evaluación}

El cuestionario LGBT-UAEH2019 se ha elaborado para esta investigación está integrado por dos secciones: la primera de la que se obtienen datos relacionados sobre la orientación sexual y la segunda relacionadas con experiencias de vida, abarcando temas como la desigualdad, la familia, entorno social, discriminación, homofobia, inclusión, reconocimiento y aceptación de sí mismo; con un total de 17 preguntas cerradas

\section{Procedimiento de Investigación}

La investigación es Cuantitativa que se mueve dentro de una ciencia nomotética cuyo objetivo es llegar a formular leyes generales, visto de otro modo, consiste en establecer relaciones causales que supongan una explicación del fenómeno. La temporalización de nuestra investigación es transversal por la elaboración de cortes estratificados de tal forma que la investigación se pueda realizar en un breve lapso de tiempo, dado la recolección de datos se realiza en un único momento. Además, es de tipo Exploratorio, porque se lleva a cabo una exploración inicial para empezar a conocer una variable o un conjunto de estas, se suele aplicar ante algún problema novedoso y constituye a una introducción a otros estudios sobre el mismo tema. Para la aplicación del instrumento se utilizó la técnica de la bola de nieve en el que intervinieron 15 estudiantes de ambas licenciaturas.

\section{Análisis de datos}

Una vez obtenidos los datos de las encuestas, se procedió a codificarlos en el programa SPSS y se obtuvieron los siguientes porcentajes:

1.- Con relación, a asumir su orientación sexual como una opción libre, un $40 \%$ de los alumnos encuestados

\section{¿Considero que es una opción la orientación sexual?}

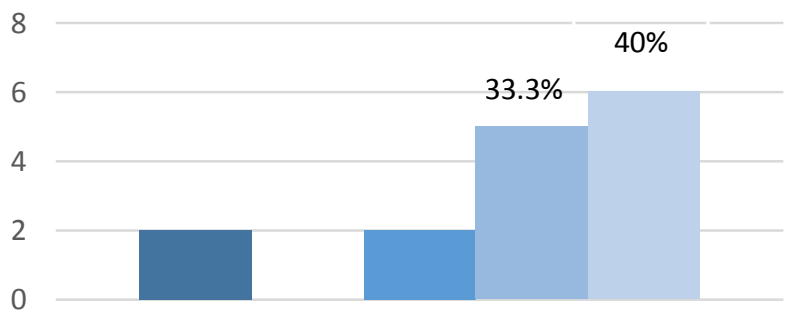

respondieron que están totalmente de acuerdo, un 33.3\% contestaron que están de acuerdo, un $13.3 \%$ tienen una opinión neutral y $13.3 \%$ contestaron que están totalmente en desacuerdo.

Gráfica 1.- Orientación sexual. Elaboración propia.

2.- Se puede distinguir que $46.7 \%$ de los alumnos tiene una actitud neutral con relación a si tuvieron dificultades para asumir su orientación sexual, mientras que $20 \%$ respondieron que están en desacuerdo, un 20\% respondieron que están de acuerdo, un $6.7 \%$ contestaron que están totalmente de acuerdo y un $6.7 \%$ están totalmente en desacuerdo.

\section{¿Tuve dificultades para asumir mi orientación sexual?}

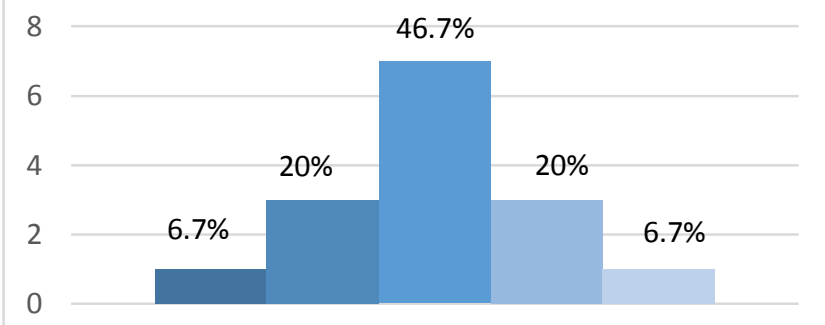

Gráfica 2.- Dificultades por la orientación sexual. Elaboración propia.

3.- Las personas que están totalmente de acuerdo en vivir su orientación sexual de manera libre se encuentra en un porcentaje del $40 \%$, un $26.7 \%$ tiene una opinión neutral, un $20 \%$ están de acuerdo y $13.3 \%$ están en desacuerdo en vivir la orientación de su sexualidad de manera libre.

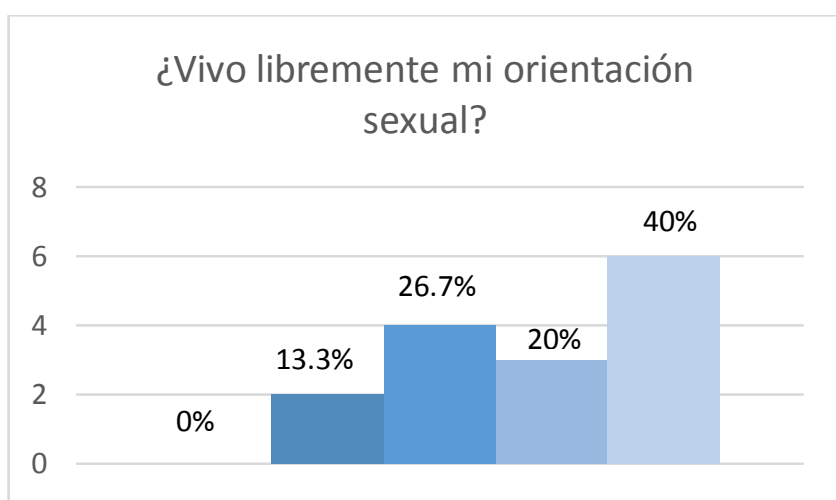

Gráfica 3.-Libertad en la orientación sexual. Elaboración propia.

4.- Con respecto a la orientación sexual sujeta a cambios, en un $60 \%$ los estudiantes tienen una opinión neutral, un $20 \%$ respondió que está totalmente de acuerdo, el $13.3 \%$ están de acuerdo y $6.7 \%$ contesto que está en desacuerdo. 
Neutral con el $60 \%$ es el porcentaje más alto, ya que los encuestados consideran que todo depende del ambiente en donde se encuentre la persona.

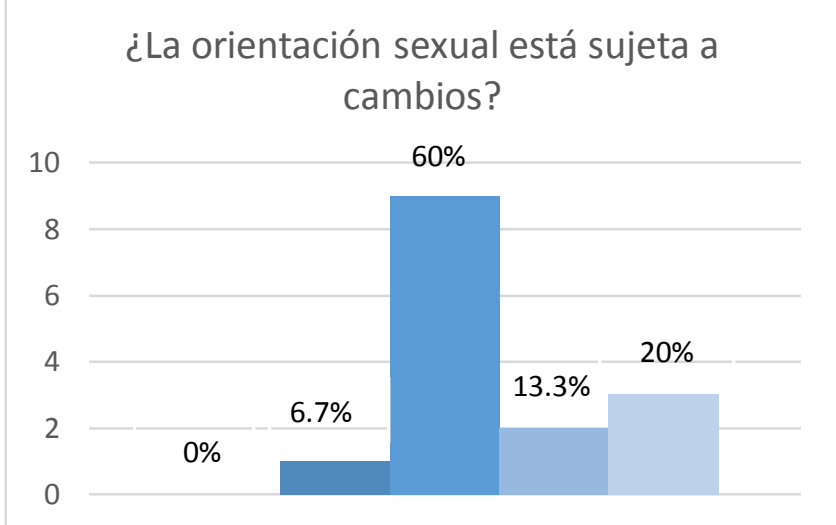

Gráfica 4.- Orientación sexual sujeta a cambios. Elaboración propia.

5.- En un $33.3 \%$ los alumnos encuestados respondieron que están en desacuerdo en relación a si ha cambiado el modo de pensar de la sociedad con respecto a diversidad de la orientación sexual, al mismo tiempo, se distingue un $33.3 \%$ de alumnos que tienen una opinión neutral, un $20 \%$ contestaron que están totalmente de acuerdo, un $6.7 \%$ que están en desacuerdo y un $6.7 \%$ están totalmente en desacuerdo.

Se muestra una postura neutral con relación a la orientación sexual con un mayor porcentaje.

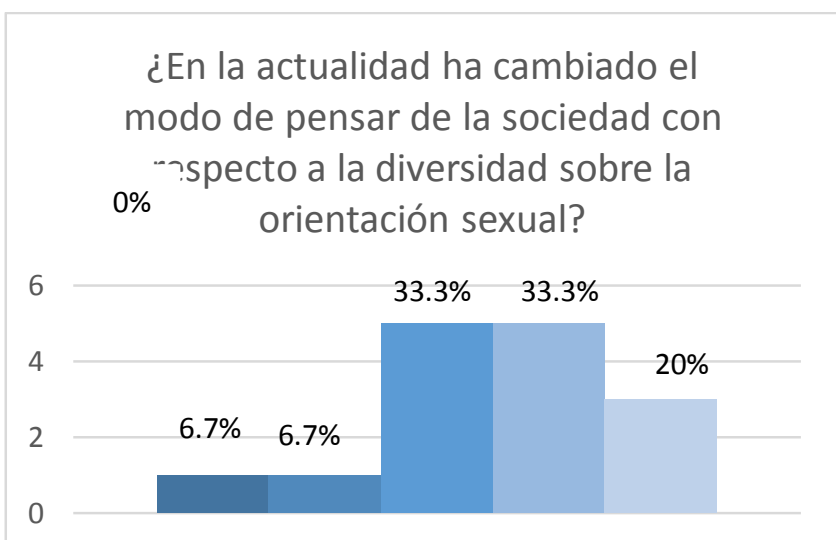

Gráfica 5.- Pensamiento de la sociedad sobre la orientación sexual. Elaboración propia.

6.- En relación, a la actitud negativa contra los homosexuales, porque los heterosexuales reprimen su sexualidad, un $40 \%$ de los estudiantes tienen una postura neutral, un $20 \%$ respondieron que están en desacuerdo, un $20 \%$ están en desacuerdo, mientras que, un $13.3 \%$ respondieron que están totalmente de acuerdo y un $6.7 \%$ están totalmente en desacuerdo.

De acuerdo es la categoría de porcentaje mayor con el $40 \%$, por lo cual se infiere que la mayoría de los estudiantes viven de manera plena su orientación sexual.

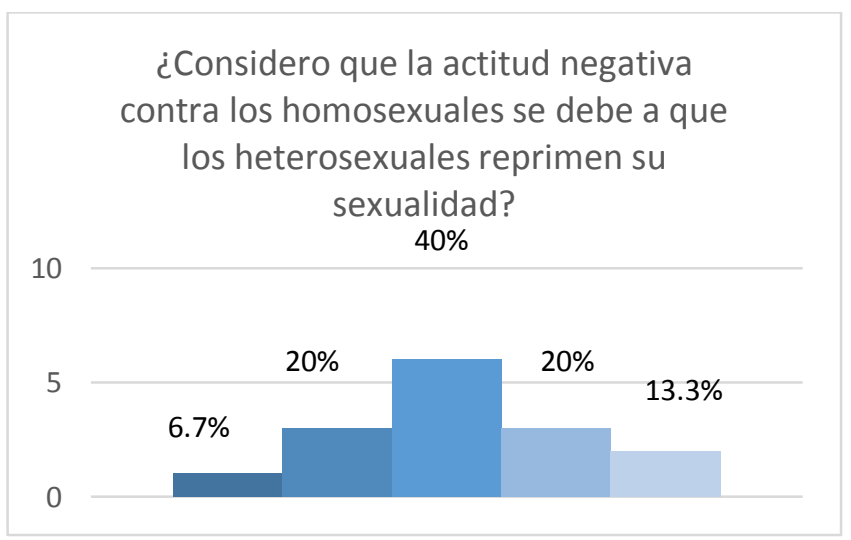

Gráfica 6.- Heterosexuales reprimen su sexualidad. Elaboración propia.

7.- Los alumnos que tienen una opinión neutral con relación a si en las parejas homosexuales hay roles de género, se encuentran en un $53.3 \%$, mientras que un $33.3 \%$ respondieron que están de acuerdo y $13.3 \%$ están en desacuerdo. Neutral es el porcentaje más alto con $53.3 \%$, debido a que la sociedad está muy acostumbrada a ver a un hombre y a una mujer en una relación.

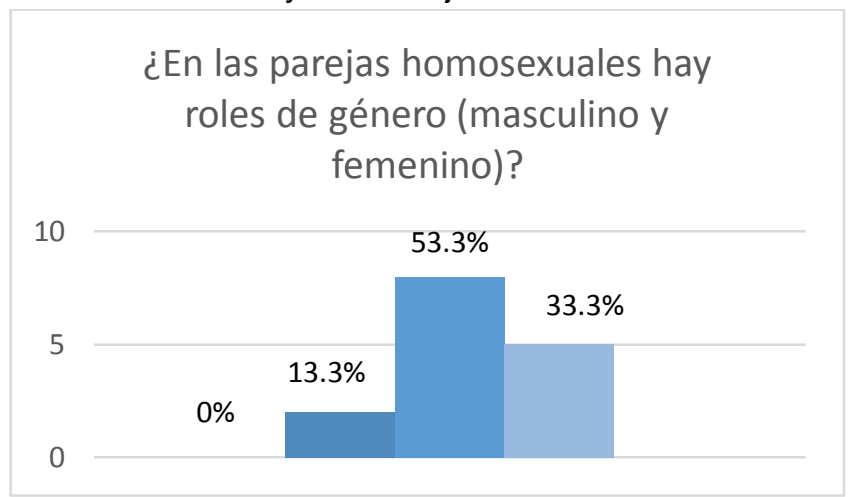

Gráfica 7.- Roles de género en parejas gay-lésbicas. Elaboración propia.

8.- En relación a los factores hormonales genéticos, desempeñan un papel importante en la elección de la sexualidad, un $40 \%$ de los estudiantes contestaron que están de acuerdo, un 33.3\% tienen una postura neutral, un $13.3 \%$ respondieron que están en desacuerdo, un $6.7 \%$ opinan que están totalmente de acuerdo y un $6.7 \%$ está totalmente en desacuerdo.

De acuerdo con el $40 \%$ es el porcentaje más alto seguido de la categoría neutra con el $33.3 \%$ esto es tal vez a que 
muchos no están informados de la investigaciones actuales.

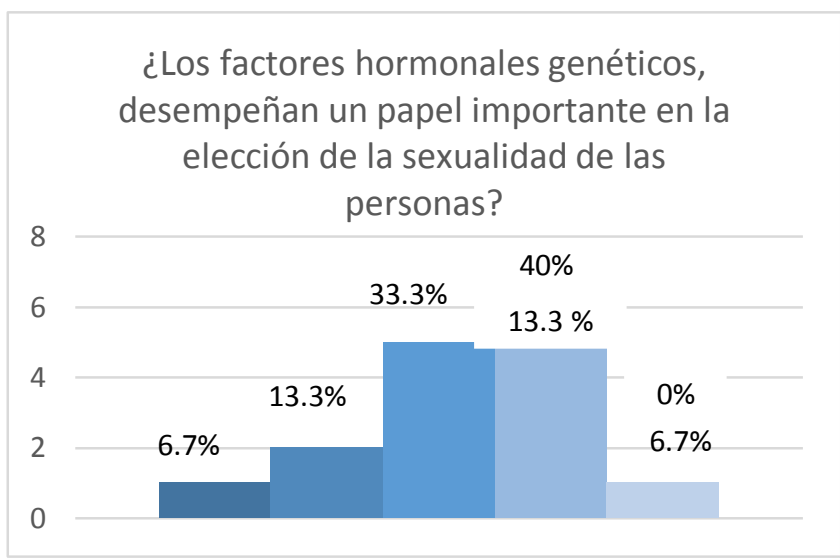

Gráfica 8.- Factores genéticos en la elección de la orientación sexual. Elaboración propia.

9.- Un $33.3 \%$ de los señalan estar de acuerdo en ser discriminados por su orientación sexual, un $26.7 \%$ opinan estar totalmente en desacuerdo un $13.3 \%$ se encuentran en una postura neutral, un $13.3 \%$ están de acuerdo y un $13.3 \%$ contestaron que están totalmente de acuerdo.

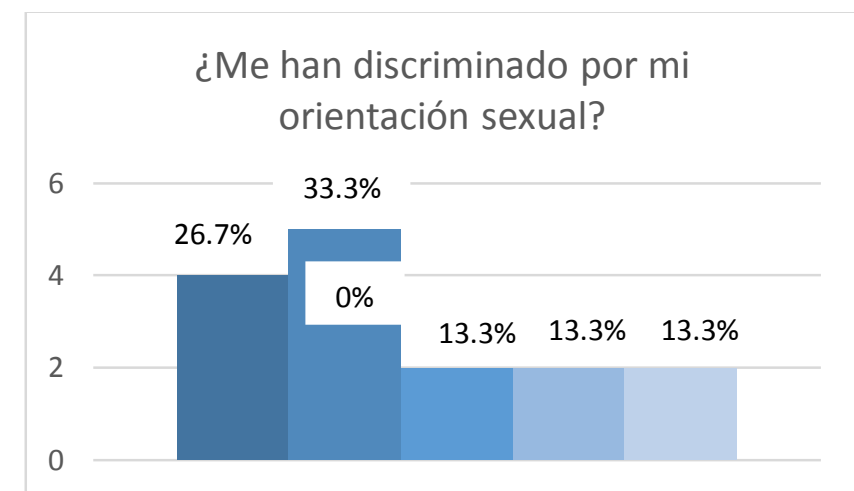

Gráfica 9.- Discriminación por orientación sexual. Elaboración propia.

10.- En un $40 \%$ los estudiantes están totalmente en desacuerdo con respecto a que se generen problemas de salud a causa de la discriminación, un $26.7 \%$ se encuentran en desacuerdo, un $20 \%$ tienen una opinión neutral y un $13.3 \%$ contestaron estar de acuerdo.

La categoría de totalmente de acuerdo es el más alto porcentaje, ya que los estudiantes consideran que la discriminación puede afectar a su salud física y emocional.

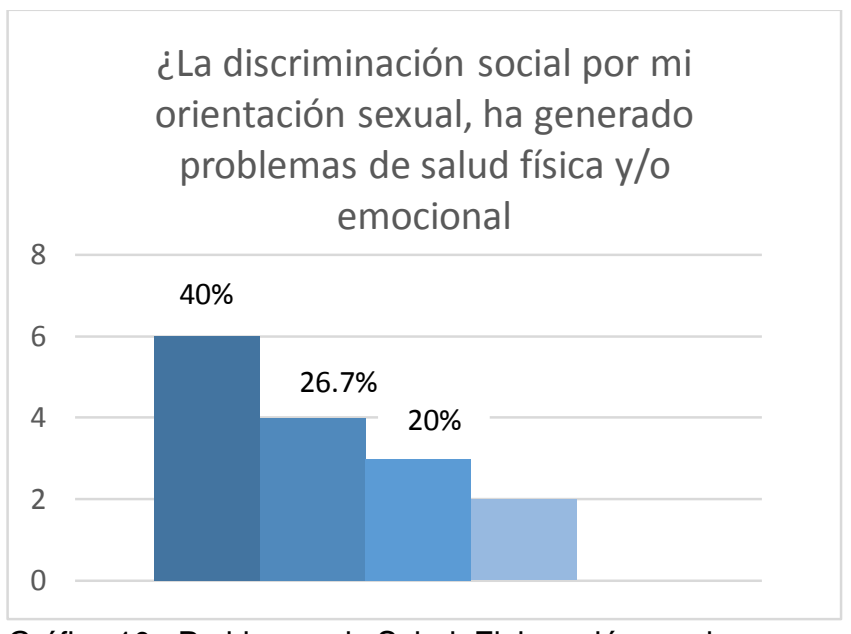

Gráfica 10.- Problemas de Salud. Elaboración propia.

11.- Un $53.3 \%$ de los estudiantes están totalmente en acuerdo sobre la importancia que reviste el apoyo de los padres con relación a la elección de su orientación sexual, un $40 \%$ se encuentra en una actitud neutral y un $6.7 \%$ respondió estar en desacuerdo.

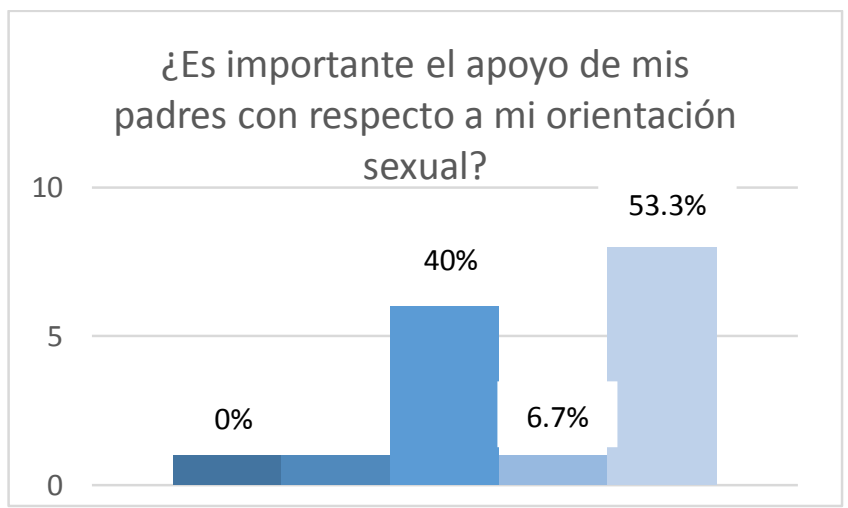

Gráfica 11.-Apoyo Familiar. Elaboración propia.

12.- Los alumnos que consideran estar de acuerdo en que les fue difícil dar conocer a su familia su orientación sexual, son un $33.3 \%$, en un $26.7 \%$ tienen una opinión neutral, un $20 \%$ respondieron estar totalmente de acuerdo, un $13.3 \%$ contestaron estar totalmente en desacuerdo solo un $6.7 \%$ opinan estar en desacuerdo. El valor de $33.3 \%$ es el más alto posiblemente por los miedos que existen hacia la discriminación hacia las diferentes orientaciones sexuales. 


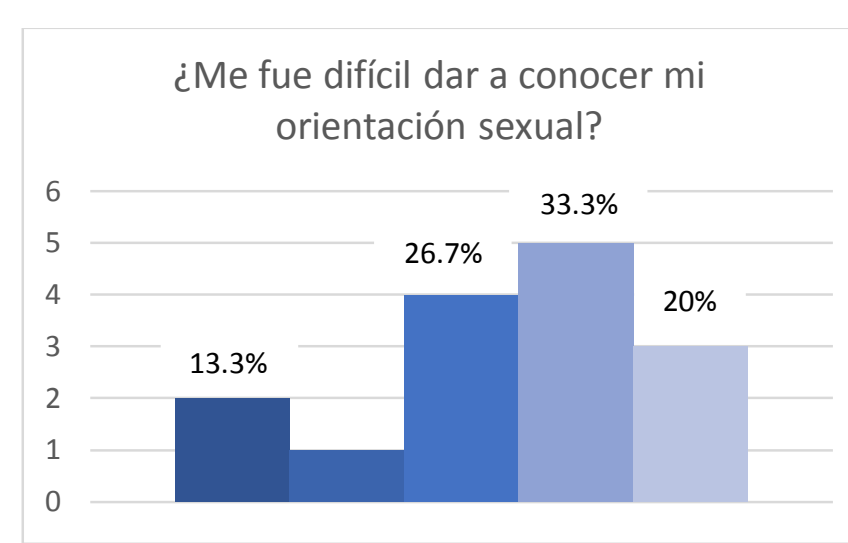

Gráfica 12.- Dificultad para dar a conocer la Orientación sexual. Elaboración propia.

13.- En relación a si ha mejorado la relación con la familia después de revelar su orientación sexual, un $40 \%$ de los estudiantes tiene una opinión neutral, un $20 \%$ de ellos respondieron estar en desacuerdo, otro $20 \%$ señalo estar totalmente de acuerdo. En un 13.3\% están de acuerdo y un $6.7 \%$ opina estar totalmente en desacuerdo.

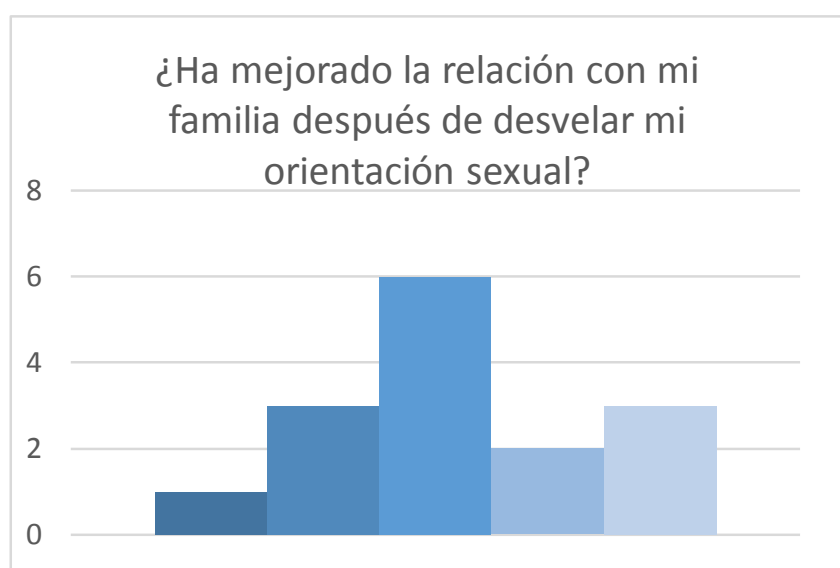

Gráfica 13.- Mejora en las relaciones familiares al revelar la Orientación sexual. Elaboración propia.

14.- En un $66.7 \%$ los estudiantes respondieron estar totalmente en desacuerdo con relación a si han pensado en el suicidio a causa de la discriminación, en un $20 \%$ respondió estar de acuerdo y un $13.3 \%$ contesto estar en desacuerdo.

El $66.7 \%$ es debido posiblemente a que los alumnos no ven al suicidio como una solución a sus problemas.

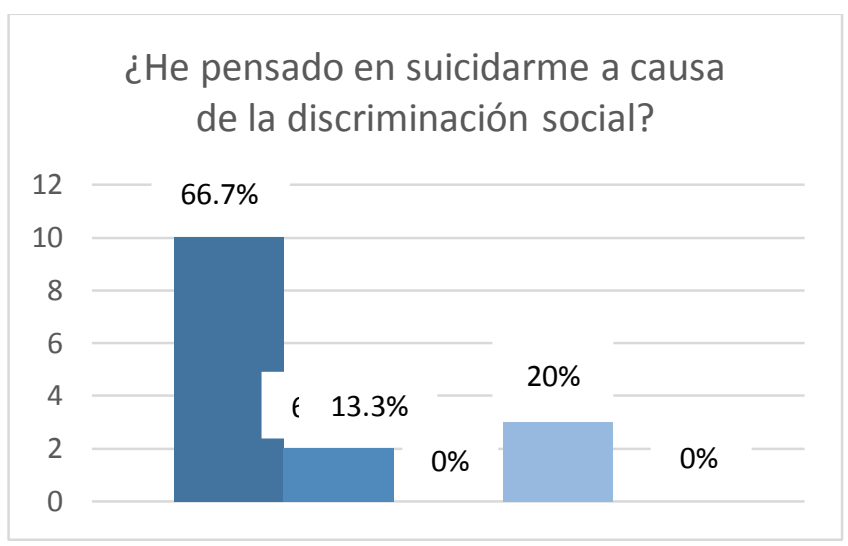

Gráfica 14.- Suicidio por discriminación. Elaboración propia.

15.- Con respecto a si han recibido rechazo de parte de sus compañeros por su orientación sexual, el $60 \%$ de los estudiantes respondieron estar totalmente en desacuerdo, un $20 \%$ tienen una opinión neutral, un $13.3 \%$ opina estar en descuerdo y un $6.7 \%$ contesto estar de acuerdo.

El $60 \%$ se debe a que la mayoría de los estudiantes reciben apoyo por parte de sus compañeros ya que se encuentran dentro de una sociedad incluyente.

$40 \%$

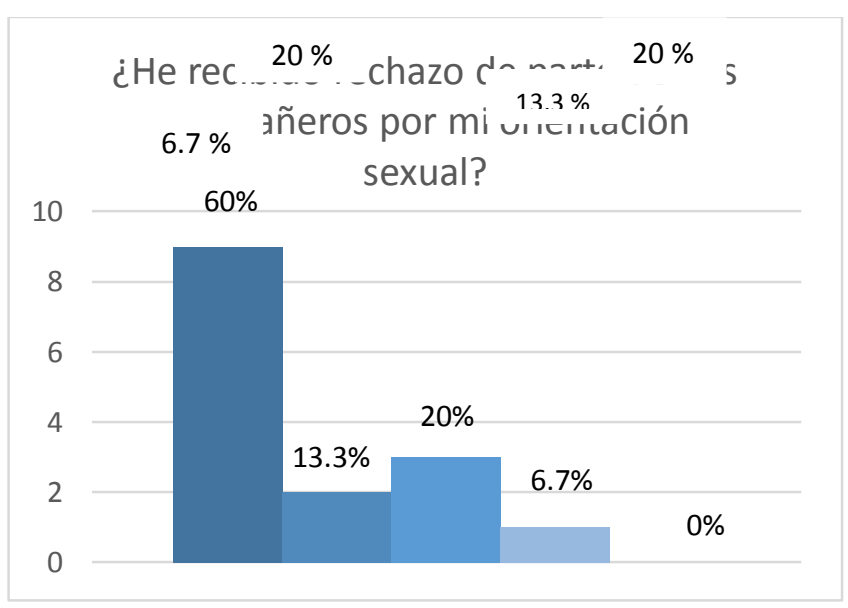

Gráfica 15.- El rechazo por la Orientación sexual. Elaboración propia.

16.- Un $26.7 \%$ de los estudiantes tiene una actitud neutral con relación a si se han sentido impotentes por la intolerancia hacia la diversidad sexual, mientras que un $20 \%$ respondieron estar en desacuerdo, otro $20 \%$ de los estudiantes señalan estar de acuerdo, un $20 \%$ están 
totalmente en desacuerdo y un $13.3 \%$ contestaron estar en desacuerdo.

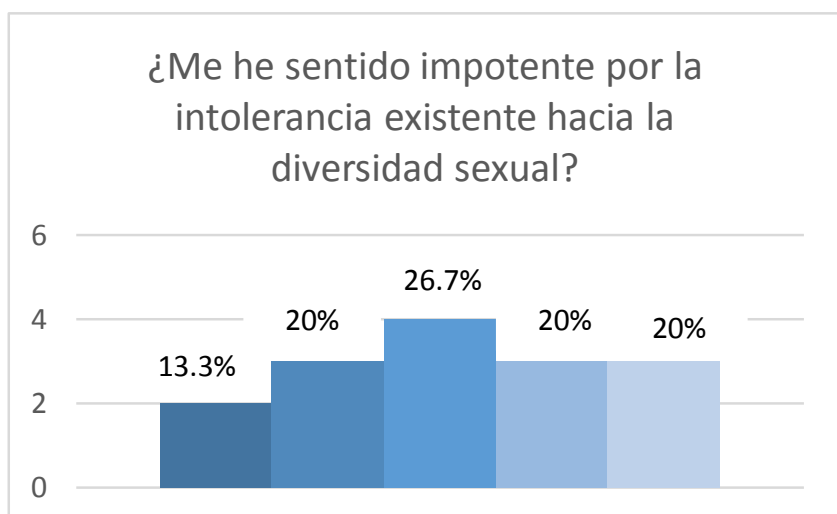

Gráfica 16.- La impotencia por la intolerancia hacia la Orientación sexual. Elaboración propia.

17.- Los estudiantes reportan estar totalmente en desacuerdo con relación a si han recibido agresión física y/o verbal a causa de su orientación sexual, en un $53.3 \%$ del total de los encuestados, mientras que un $20 \%$ respondieron estar en desacuerdo, un $13.3 \%$ consideran estar de acuerdo y otro $13.3 \%$ contestaron estar totalmente de acuerdo.

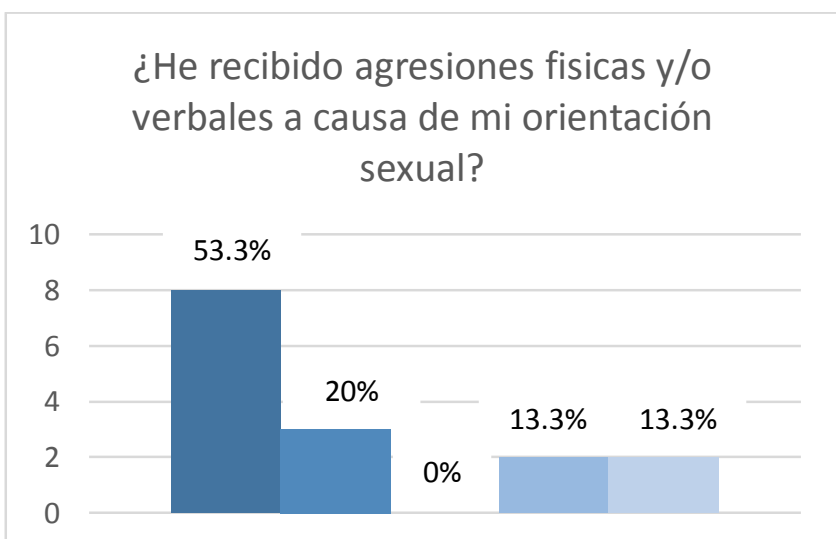

Gráfica 17.-Agresiones físicas y/o verbales por la Orientación sexual. Elaboración propia.

\section{Análisis de Resultados}

La participación de los estudiantes de la Escuela Superior de Actopan en esta investigación permitieron que los resultados de las encuestas se generalicen y se asevere que los estudiantes encuestados vivan de manera plena su orientación sexual, anteriormente una persona que decidía ser parte de la comunidad LGBT inmediatamente era mal visto y principalmente rechazado por su familia como consecuencia se generaban problemas de salud mental a tal grado de llegar a pensar y culminar en el suicidio, también recibían agresiones físicas o verbales por la elección de la orientación sexual. Sin embargo, varios homosexuales y bisexuales siguen sin desvelar su orientación, debido a la presión social sobre "lo que espera ver la sociedad en ellos" es una carga que día a día no resulta fácil de llevar, con el paso del tiempo se ha trasformado la concepción de las personas con orientación sexual diferente a la heterosexual, en esta investigación se encontró que los alumnos señalan no recibir rechazo de parte de los compañeros, al contario ellos se encuentran dentro de una sociedad incluyente en la que no son rechazados, se puede señalar que el hecho de estudiar en una escuela pública los estudiantes tienen una mentalidad más abierta, libre de prejuicios. Los alumnos encuestados consideraron que es importante el apoyo familiar, la expresión de amor y aceptación de parte de sus familiares y amigos para el bienestar como personas. La mayoría afirmo tener dificultad para dar a conocer a su familia su elección sobre la orientación sexual, debido a la reacción que tienen los padres que será de gran impacto en su vida, implica un gran esfuerzo el revelarlo, valor y respeto son las formas de afrontamiento que consideran más adecuadas para desvelar su orientación sexual. Por otra parte, ellos no han tomado en cuenta el suicidio para la solución a sus problemas, solamente dos personas afirmaron estar de acuerdo, probablemente no se tiene el apoyo de los familiares y se prefiere ocultarlo. En la comunidad estudiantil, algunos alumnos asumieron una postura neutra sobre su orientación sexual, reconociendo que nos encontramos en pleno siglo XXI, donde la sociedad acepta y respeta como tal la homosexualidad; gracias a la visibilidad que ha tenido el movimiento LGBT y el logro de grandes avances sociales y políticos: cada año va aumentando el número de personas que participan en actividades, en las marchas y el fenómeno que se presenta es permitir la tolerancia en muchos espacios públicos, esto no quiere decir que todos los acepten, existen personas que no piensan igual, aún subsiste la homofobia, depende de cada persona la concepción que tenga sobre este fenómeno social y dependerá de esto su forma de actuar ante estas situaciones; en México se han dado pasos para el reconocimiento de los derechos de las parejas homosexuales, se aprobó la reforma en Hidalgo precisamente en este año 2019, que permite visualizar que la inclusión ha mejorado en estos espacios así como la visibilidad en zonas urbanas.

Con respecto a la frase "considero que la actitud negativa contra los homosexuales es porque los heterosexuales reprimen su sexualidad" se presenta un estado neutral y de acuerdo, es posible que algunos oculten su orientación sexual y por ende sigan sin desvelarla, son pocos los 
casos de personas reprimidas que pudimos observar en esta investigación, sin embargo, esta la contraparte que explica mejor la homofobia, más allá del hecho de tratar de reprimir el deseo homosexual. De igual manera, los jóvenes respondieron a un estado neutral al preguntarles si hay roles de género en una pareja no heterosexual, llegando a la conclusión de que habrá probabilidad de que existan estos roles, ya que la sociedad está muy acostumbrada a ver a un hombre y a una mujer en una relación, aunque algunas parejas si lo consideran y lo ponen en práctica, en otras no tendrá ninguna importancia. Con relación a la opinión de los demás, si es cierto que la orientación sexual está sujeta a cambios, respondieron no estar de acuerdo ni en descuerdo, porque todo depende del ambiente en donde se encuentre la persona, en caso de tener dudas desde antes sobre su preferencia, y durante un proceso la persona se dará cuenta sobre donde quiere estar. La genética desempeña un papel importante en la elección de la sexualidad, muchos contestaron estar de acuerdo y también algunos tienen una opinión neutral, tal vez muchos no estén informados, algunos estudios han revelado que un cromosoma de más en su genética provoca cambios en su cuerpo físico además algunos investigadores han mostrado que la homosexualidad es más común en hermanos y parientes en la misma línea materna y existe un factor genético como causa. Esta es la parte en donde se hace hincapié para cuestionarse sobre: ¿se nace o se hace?, aún no se sabe con certeza, pero es un gran tema a discusión que en algunos años más la ciencia desvelará con respecto al estudio de los genes.

\section{Conclusión}

El reconocer que la mayor parte de los fenómenos que se generan dentro de una sociedad son producto de una construcción social compleja, nos permite ver que el individuo es el reflejo de la sociedad y no de sí mismo, en el caso de la homosexualidad y bisexualidad algunos reprimen su homosexualidad y no se habla de este tema abiertamente, temen al rechazo, discriminación y violencia que pueden presentarse debido a su orientación sexual, incluso son mal vistos, se les ve con odio o con lástima en la mejor de las ocasiones y en el proceso de dar a conocer su orientación sexual pueden perder a sus familiares, amistades o personas queridas que pensaban en algún momento los aceptarían al desvelar su orientación sexual. La homofobia se caracteriza por la violación de los derechos humanos, la violencia física y verbal hacia ellos; está condición está implícita en donde los heterosexuales pueden mostrarse amables con ellos, pero en determinadas circunstancias pueden negar este derecho. En algunas circunstancias el rechazo es abierto o también se oculta. El rechazo subsiste entre los jóvenes universitarios, solo que es sutil, se ve a distancia a los estudiantes con una preferencia distinta, pero el fenómeno sigue presente.

A pesar de que México se ha integrado a la sociedad poco a poco la homosexualidad (González, R., \& Valles, R., 2010), algunos espacios son más marcados que en otros, se les acepta si no se exhiben públicamente y no muestren conductas inapropiadas. La percepción de la población hacia la homosexualidad o lesbianismo es distinta, entre hombres hay más rechazo hacia la orientación homosexual que en mujeres, esto provoca que la comunidad homosexual sea más vulnerable ante una sociedad machista.

El desvelar la orientación sexual implica enfrentarse a mayor estrés psicosocial por el rechazo social a su preferencia, la incomprensión conlleva un mayor riesgo de exposición a enfermedades como: anorexia, bulimia, depresiones, ansiedad, consumo y abuso de sustancias y a un incremento de intentos de suicidio. Los profesionistas encargados de la psique, deben estar preparados para manejar estos problemas, así como orientar a los padres en la respuesta hacia la sexualidad de sus hijos, esto presentará menores niveles de homofobia y mejorará el ajuste psicosocial que también prevendría daños a la salud mental (Granados, J, Y Delgado, G., 2008).

\section{Referencias}

[1] Careaga, G. C. (2004). La familia, apoyo y represión para el ejercicio de la sexualidad. En C. Gloria, \& S. Cruz, Sexualidades diversas. (págs. 2003-2015). México: Universidad Nacional Autónoma de México.

[2] Fregoso, J. (09 de Junio de 2018). Acerca de nosotros: Infobae. Obtenido de Infobae:

https://www.infobae.com/america/mexico/2018/06/09/gaysindigenas-y-de-piel-morena-una-triple-pesadilla-dediscriminacion-en-mexico/

[3] Gasque, M. (2016). Freud y la homosexualidad. Desde el diván, pág. 265-271.

[4] González, R., y Valles, R. (2010). La construcción de identidades homosexuales en México: Un caso de discriminación, intolerancia, violencia y estigmatización en un grupo de jovenes urbanos. UAEH, 1-16.

[5] Granados, J, Y Delgado, G. (2008). Indentidad y riesgos para la salud mental de jovenes gays en México: recreando la experiencia homosexual. ARTIGO, 24(5), 1042-1050.

[6] Hernandez, R. F. (2006). Metodologia de la Investigación. México: Mc Graw Hill.

[7] Hoy, T. (2013). Sabias que todos nacemos Bisexuales. tabasco hoy. Obtenido de https://www.tabascohoy.com/nota/160268/sabiasque-todos-nacemos-bisexuales

[8] Jiménez, A., y Romero, M. (2014). "Salir del closet" en la Ciudad de México. Scielo, 37(5), 391-397.

[9] Lazano, I. (2009). El significado de homosexualidad en jóvenes de la ciudad de México. Enseñanza e Investigación en Psicología, 153-168.

[10] Mansera, J. (2012). Prejuicio, diversidad sexual y religión. CRITERIOS, 55-76. 
[11] Moral, J. (2011). Homosexualidad en la juventud mexicana y su distribución geográfica. CIEAP/UAEM, No. 67.

[12] OPS/OMS. (2011). Día internacional contra la homofobia: 17 de mayo del 2011. Obtenido de

https://www.paho.org/hq/index.php?option=com_content\&vie $\underline{\mathrm{w}=\text { article } \& i d=5411: 2011 \text {-dia-internacional-contra-homofobia- }}$ 17-mayo-2011\&Itemid=1926\&lang=es

[13] Piña, J., \& Aguayo, H. (2015). Homofobia en estudiantes universitarios de México. Región y sociedad., 5-35.

[14] PND/PGA. (2017). Promoviendo los derechos humanos y la inclusión de la personas LGBTI: un manual para los parlamentarios y las parlamentarias. Empowered lives, 1-84.

[15] Pérez, B. (2005). Homosexualidad: Secreto de familia. El manejo del secreto en familias con algún miembro homosexual. Madris: EGALES.

[16] Redondo, M. (13 de Enero de 2017). Acerca de nosotros: hipertextual. Obtenido de hipertextual: https://hipertextual.com/2017/01/homosexualidad-mexico

[17] Richaed, A. (2013). Lgbt: Con el mismo derecho a los derechos humanos y la dignidad. Orientación sexual e identidad de género y la protección de los migrantes forzados. Obtenido de: https://www.fmreview.org/es/osig/richard

[18] Troiden, R. (1989). The formation of Homosexual identities. J Homosexuality; 17: 43-74.

[19] Uribe, M., Pérez, E., y Arotoma, R. (2018). Actitudes de los padres hacia la homosexualidad de sus hijos. HORIZONTE DE LA CIENCIA, 8(15), 71-81. doi:2304-4330

[20] Vera, L. (1998). Historia de la sexualidad. Biomedic, 9(2), 116-121.

[21] Vitalit, J., \& Onofrio, R. (2011). Salir del closet: La aceptación del encuentro con uno. Asociación Argentina de Salud Mental., 111. 